Acta Psychologica xxx (2013) xxx

Memory for symmetry and perceptual binding in patients with schizophrenia

Vincenzo Cestari ${ }^{\mathrm{a}, \mathrm{b}}$, Daniele Saraulli ${ }^{\mathrm{a}, \mathrm{b}}$, Pietro Spataro ${ }^{\mathrm{a}}$, Alessandro Lega ${ }^{\mathrm{c}}$, Antonio Sciarretta ${ }^{\mathrm{c}}$, Valéria Rezende Marques ${ }^{\mathrm{a}, \mathrm{d}}$, Clelia Rossi-Arnaud ${ }^{\mathrm{a}, *}$

a Department of Psychology, Sapienza University of Rome, Via deiMarsi 78, 00185 Rome, Italy

${ }^{b}$ Cell Biology and Neurobiology Institute, C.N.R. National Research Council of Italy, Via del Fosso di Fiorano 64/65, 00143 Rome, Italy

c Acute Psychiatric Care Unit, Department of Mental Health RM-G, San Giovanni Evangelista Hospital, Via Antonio Parrozzani 3, 00019 Tivoli, Italy

d CAPES Foundation, Ministry of Education of Brazil, Setor Bancário Norte, Quadra 2, Bloco L, Lote 06, CEP 70040-020 Brasília, DF,Brazil

- We examined perceptual binding in schizophrenic patients.

- Participants recalled symmetrical and asymmetrical patterns of increasing length.

- Binding deficits in patients could not be ascribed to lower visuospatial ability.

- Impairment in patients was magnified with supercapacity patterns.

- Patients were more likely to recall symmetrical patterns as asymmetrical. 


\title{
1 Memory for symmetry and perceptual binding in patients
} 2 with schizophrenia

\author{
Q1 Vincenzo Cestari ${ }^{\mathrm{a}, \mathrm{b}, 1}$, Daniele Saraulli ${ }^{\mathrm{a}, \mathrm{b}, 1}$, Pietro Spataro ${ }^{\mathrm{a}, 1}$, Alessandro Lega ${ }^{\mathrm{c}}$, Antonio Sciarretta ${ }^{\mathrm{c}}$, \\ Valéria Rezende Marques ${ }^{\mathrm{a}, \mathrm{d}}$, Clelia Rossi-Arnaud ${ }^{\mathrm{a}, *}$ \\ a Department of Psychology, Sapienza University of Rome, Via deiMarsi 78, 00185 Rome, Italy \\ b Cell Biology and Neurobiology Institute, C.N.R. National Research Council of Italy, Via del Fosso di Fiorano 64/65, 00143 Rome, Italy \\ c Acute Psychiatric Care Unit, Department of Mental Health RM-G, San Giovanni Evangelista Hospital, Via Antonio Parrozzani 3, 00019 Tivoli, Italy \\ d CAPES Foundation, Ministry of Education of Brazil, Setor Bancário Norte, Quadra 2, Bloco L, Lote 06, CEP 70040-020 Brasília, DF,Brazil
}

\section{A R T I C L E I N F O}

\section{Article history:}

Received 17 January 2013

Received in revised form 24 September 2013

Accepted 27 September 2013

Available online $\mathrm{xxxx}$

\section{PsycINFO classification code:}

2343

Learning \& Memory

Keywords:

Schizophrenia

Binding

Symmetry

Memory

\begin{abstract}
A B S T R A C T
The present study investigated the use of perceptual binding processes in schizophrenic (SC) patients and 28 matched healthy controls, by examining their performance on the recall of symmetrical (vertical, horizontal 29 and diagonal) and asymmetrical patterns varying in length between 2 and 9 items. The results showed that, al- 30 though SC patients were less accurate than controls in all conditions, both groups recalled symmetrical patterns 31 better than asymmetrical ones. The impairment of SC patients was magnified with supra-span symmetrical ar- 32 rays, and they were more likely to reproduce symmetrical patterns as asymmetrical, particularly at medium 33 and high length levels. Hierarchical regression analyses further indicated that the between-group differences 34 in the recall of supra-span vertical and horizontal arrays, which require a greater involvement of visual pattern 35 processes, remained significant after removing the variance associated with performance on asymmetrical pat- 36 terns, which primarily reflects intrafigural spatial processes. It is proposed that schizophrenia may be associated 37 with a specific deficit in the formation and retrieval of the global visual images of studied patterns and in the use 38 of the on-line information about the type of symmetry being tested to guide retrieval processes.
\end{abstract}

(c) 2013 Elsevier B.V. All rights reserved. 40

\section{Introduction}

Memory dysfunctions have been widely documented in patients with schizophrenia (SC), both in long-term memory (LTM) and in workingmemory (WM) tasks (Aleman, Hijman, Haan, \& Kahn, 1999; Forbes, Carrick, McIntosh, \& Lawrie, 2009; Lee \& Park, 2005). The concept of WM refers to a limited capacity system for the temporary storage and manipulation of information. According to the latest model proposed by Baddeley (2012), WM consists of four separate subsystems: (a) the phonological loop, which maintains verbal traces; (b) the visuospatial sketchpad, which retains visual and spatial stimuli; (c) the central executive, which supervises ongoing processing; and (d) the episodic buffer, defined as a multidimensional store allowing features from different sources, including perceptual input and LTM knowledge, to be bound into chunks or episodes (Baddeley, 2000; Baddeley, Allen, \& Hitch, 2011). In recent years, the issue of binding has been the focus of a large body of research. Here, we will refer to a widely accepted distinction

\footnotetext{
* Corresponding author. Tel.: + 3906 49917513; fax: + 390649917711.

E-mail addresses: vincenzo.cestari@ipsifar.rm.cnr.it (V. Cestari)

daniele.saraulli@ipsifar.rm.cnr.it (D. Saraulli), pietro.spataro@uniroma1.it (P.Spataro), alega@tiscali.it (A. Lega), an.sciarretta@libero.it (A. Sciarretta),

valeriarsmarques@gmail.com (V.R. Marques), clelia.rossi-arnaud@uniroma1.it (C. Rossi-Arnaud).

1 These authors contributed equally to the manuscript.
}

between controlled and perceptual binding processes (Allen, Baddeley, \& 61 Hitch, 2006; Allen, Hitch, \& Baddeley, 2009; Karlsen, Allen, Baddeley, \& 62 Hitch, 2010; Mitchell, Johnson, Raye, Mather, \& D'Esposito, 2000). 63

Controlled (or active) binding processes refer to the integration of 64 unrelated items or different object features, such as form and colour 65 (Treisman, 2003), or to the association of objects to their spatial posi- 66 tions (Elsley \& Parmentier, 2009). For instance, a pair of words such as 67 "elephant" and "umbrella" can be remembered by forming a mental 68 image of an elephant holding the umbrella over its head (Allen et al., 69 2009; Baddeley, 2007). Most studies investigating this type of binding 70 have asked participants to recall the identity and the spatial position 71 of alphabetical letters located in a $3 \times 3$ matrix (Burglen et al., 2004; 72 Mitchell et al., 2000) or to recognise colour-shape combinations in a 73 change detection task (Allen, Hitch, Mate, \& Baddeley, 2012; Luck \& 74 Vogel, 1997; Treisman \& Zhang, 2006).

On the other hand, perceptual binding processes (also called percep- 76 tual grouping: Treisman, 2003) are thought to underlie visuospatial or 77 verbal tasks in which individual items can be grouped together in mem- 78 ory on the basis of bottom-up Gestalt principles (e.g., proximity, symme- 79 try and connectedness: Woodman, Vecera, \& Luck, 2003) or LTM 80 semantic knowledge. One of the major challenges leading to the proposal 81 of the episodic buffer (Baddeley, 2000) was the need to account for the 82 interaction between WM and LTM linguistic and visuospatial knowledge. 83 In a series of experiments, Jefferies, Lambon Ralph, and Baddeley (2004) 84 
and later Baddeley, Hitch, and Allen (2009) examined the attentional requirements underlying memory for prose and sentences. According to the revised model of WM (Baddeley, 2000, 2012), the recall of meaningful sentences and prose passages cannot be accounted for by the phonological loop alone, because of its inherent capacity limits. Instead, the assumption is that memory span for prose and sentences can be enhanced by the interaction between the phonological loop and LTM semantic and linguistic knowledge (i.e., long-term representations involved in the comprehension of the theme and the overall meaning of sentences), which would result in the formation of larger, integrated chunks in the episodic buffer (Baddeley et al., 2009). Both studies found that demanding secondary tasks reduced the overall recall performance, but did not eliminate the memory advantage of sentences and prose passages (relative to sequences of unrelated words and meaningless passages), suggesting that the integration of phonological and long-term linguistic information is not attention-demanding per se (Jefferies et al., 2004). Along the same line of work, a number of studies have compared the recall of symmetrical and asymmetrical patterns in healthy participants (Imbo, Szmalec, \& Vandierendonck, 2009; Kemps, 2001; Pieroni, Rossi-Arnaud, \& Baddeley, 2011; Rossi-Arnaud, Pieroni, \& Baddeley, 2006; Rossi-Arnaud, Pieroni, Spataro, \& Baddeley, 2012). In short-term memory, the typical finding is that visuospatial performance is significantly better for vertical than for asymmetrical configurations. Paralleling the findings obtained with sentences, twice as many spatial elements can be recalled when they are arranged to form a vertically symmetrical pattern than when they form an asymmetric configuration (at least with a simultaneous presentation: Rossi-Arnaud et al., 2012). The WM model (Baddeley, 2000, 2012) suggests that such an increase in memory span is brought about by an interaction between the visuospatial sketchpad and the LTM knowledge about the configurational properties of symmetrical arrays. In particular, healthy adults presented with symmetrical patterns have been found to use axis-based retrieval strategies, like side-side reflection (i.e., constructing one side of the array and then completing the other side by reflection across the symmetry axis) and point-for-point correspondence (i.e., placing pairs of dots in point-forpoint correspondence across the symmetry axis), which would imply the storage in the episodic buffer of a lower number of integrated chunks (Bornstein \& Stiles-Davis, 1984). The main result from the aforementioned studies is that the advantage of vertical symmetry was not eliminated by a range of secondary tasks aimed at selectively interfering with the activity of the phonological loop (articulatory suppression: Rossi-Arnaud et al., 2006, 2012), the visuospatial sketchpad (sequential tapping: Kemps, 2001; Pieroni et al., 2011; Rossi-Arnaud et al., 2012) and the central executive (the verbal-trail task: Rossi-Arnaud et al., 2006, 2012). Based on these data, Rossi-Arnaud et al. (2006), and later Karlsen et al. (2010), concluded that the higher performance with vertical paths reflected perceptual binding processes occurring at a preattentive stage and largely independent of executive resources.

Previous studies have begun to examine the functioning of controlled and perceptual binding processes in SC patients. Regarding controlled binding, the results have been controversial. Although Burglen et al. (2004) and Salamé, Burglen, and Danion (2006) found a disproportionate deficit in the combination condition of an object-location binding task, other researchers reported equal impairments in the single-feature and binding conditions (Luck, Buchy, Lepage, \& Danion, 2009), and suggested that the altered performance of SC patients for bound features might be the consequence of a more general reduction in visuospatial span (Luck, Foucher, Offerlin-Meyer, Lepage, \& Danion, 2008) or of attentional deficits in the ability to selectively encode information for WM storage (Gold, Wilk, McMahon, Buchanan, \& Luck, 2003). A number of studies have also been conducted to examine perceptual binding in SC patients (Silverstein, Bakshi, Chapman, \& Nowlis, 1998; Silverstein, Bakshi, Nuernberger, Carpinello, \& Wilniss, 2005). Overall, the general consensus is that the processing of stimuli with "prepotent" configurational structures is spared in SC patients, and that their deficits in perceptual organizational reflect difficulties of consolidation and/or a failure in the development of top-down response 151 strategies (Silverstein et al., 2006). Symmetry is considered to be a fun- 152 damental, early developing and possibly innate visual property to which 153 the perceptual system is predisposed (Bornstein \& Krinsky, 1985; 154 Bornstein \& Stiles-Davis, 1984; Fisher, Ferdinandes, \& Bornstein, 155 1981). The available evidence indicates that SC patients are not signifi- 156 cantly impaired in the perceptual elaboration of symmetrical patterns 157 and that they are able to use symmetry to enhance their memory per- 158 formance (Knight, Manoach, Elliott, \& Hershenson, 2000; see Uhlhaas 159 \& Silverstein, 2005, for a review), as indicated by superior short-term 160 recognition of highly structured compared to unstructured visual pat- 161 terns (Silverstein et al., 1998, 2005).

The aims of the present study were fourfold. The first and more 163 general purpose was to ascertain whether SC patients (like healthy con- 164 trols) recalled symmetrical patterns significantly better than asymmet- 165 rical ones, which would indicate that they retain the ability to bind the 166 long-term information about symmetry with the short-term content of 167 the visuospatial sketchpad (Baddeley, 2007; Imbo et al., 2009; Kemps, 168 2001). This was achieved by comparing the overall accuracy of the 169 two groups in the recall of symmetrical (vertical, horizontal and diago- 170 nal) and asymmetrical patterns varying in length between 2 and 9 items 171 (Pieroni et al., 2011; Rossi-Arnaud et al., 2006, 2012). Based on previous 172 results (Silverstein et al., 1998, 2005), we predicted that, in the analysis 173 of the overall performance (collapsed across all length levels), SC pa- 174 tients would show lower recall accuracy than healthy controls on all 175 types of patterns (symmetrical and asymmetrical), since they have 176 been found to suffer a reduction in the number of items that can be 177 stored in WM (Gold et al., 2010); however, both groups should exhibit 178 the typical recall advantage of symmetrical over asymmetrical patterns 179 (Silverstein et al., 1998, 2005).

In addition to this general analysis, we aimed at examining in more 181 details the mechanisms involved in the recall of studied patterns. The 182 between-group differences in the overall accuracy cannot provide use- 183 ful information about this issue, for two reasons: a) because the perfor- 184 mance is collapsed across all length levels, making it difficult to 185 determine the influence of such a variable on the performance of con- 186 trols and SC patients; and b) because symmetrical and asymmetrical ar- 187 rays rely to a different extent on spatial and visual processes. Therefore, 188 the second aim of our study was to verify whether the impairment of SC 189 patients was magnified when they were confronted with supercapacity 190 arrays. For the present purposes, super-capacity patterns were defined 191 as those comprising 5 or more items, whereas sub-capacity patterns 192 contained 4 or less items. This choice is consistent with the notion 193 that the capacity limit of visuospatial WM is about four integrated ob- 194 jects (i.e., chunks; Cowan, 2001; Luck \& Vogel, 1997). We expected to 195 find significant differences between SC patients and controls in the re- 196 call of symmetrical patterns at high, but not at low memory loads, 197 since Gold et al. (2003) reported that the decrease in performance 198 from set size 4 to set size 6 was greater for SC patients than for matched 199 healthy adults.

As concerns the role of visual and spatial processes, Lecerf and de 201 Ribaupierre (2005) proposed that three independent mechanisms 202 would be simultaneously recruited when recalling visuospatial arrays 203 such as those employed in the present study: extrafigural spatial processes 204 (responsible for anchoring the pattern with respect to an external frame 205 of reference), intrafigural spatial path processes (responsible for retrieving 206 the spatial relations between individual items) and intrafigural visual pat- 207 tern processes (responsible for remembering the arrays as integrated, 208 global configurations). Lecerf and de Ribaupierre (2005) found higher 209 recognition memory with a simultaneous rather than with a sequential 210 presentation, and attributed this result to the fact that, in addition to 211 the extrafigural and intrafigural path processes (recruited in both condi- 212 tions), the simultaneous presentation would also involve the formation 213 of global visual images of the to-be-remembered patterns. In a later 214 study, using procedures and stimuli similar to those employed in the 215 present study, Rossi-Arnaud et al. (2012) showed that vertically, 216 
horizontally and diagonally symmetric patterns were better recalled in the simultaneous than in the sequential presentation, whereas asymmetrical patterns were recalled equally well in the two conditions. This lack of effect of presentation modality suggests that the three processes postulated by Lecerf and de Ribaupierre (2005) contribute to a different degree to short-term memory for asymmetric and symmetric configurations. That is, the recall of irregular arrays is heavily based on extrafigural and intrafigural path processes, whereas the recall of symmetric patterns requires a more extensive recruitment of visual pattern processes. Hence, the third aim of the present study was to assess the differential contribution of these three processing modes and ascertain whether SC patients have a selective deficit in the retrieval of the global visual images of studied patterns. To this purpose, we employed a multiple regression procedure illustrated by Lorsbach and Reimer (2005) (see the Results section).

Finally, the fourth aim was to examine the type of errors produced by SC patients when recalling symmetrical patterns. This analysis can provide important clues about the question of whether SC patients are impaired in the retrieval of the global visual images of symmetrical patterns and, more generally, about the nature of SC-related deficits (Brébion, David, Jones, Ohlsen, \& Pilowsky, 2005, 2007; Brébion, Gorman, Malaspina, \& Amador, 2005; Elvevag, Weinberger, \& Goldberg, 2001; Lee, Folley, Gore, \& Park, 2008). In agreement with the theoretical framework proposed by Lecerf and de Ribaupierre (2005), in the present study we distinguished between three types of errors that can be attributed to deficits in extrafigural, intrafigural path or intrafigural pattern processes (see the Method section for a detailed description). If SC patients are impaired in the formation and retrieval of the global visual images of studied patterns, they should make significantly more intrafigural pattern errors (relative to healthy controls), particularly when recalling super-capacity symmetrical patterns.

In summary, the present study aimed at providing new evidence concerning the question of whether SC patients are able to use Gestalt principles like symmetry to boost their memory performance. In particular, we were interested in: a) comparing the overall performance of SC patients and age-matched controls on the recall of symmetrical and asymmetrical patterns; b) assessing whether increases in the length of symmetrical and asymmetrical patterns had larger detrimental effects on SC patients than on healthy controls; c) determining, through the use of hierarchical regression analysis, whether SC patients were significantly impaired in the retrieval of the global visual images of symmetrical patterns, independently from their deficits in the use of (spatial) extrafigural and intrafigural path processes; and d) ascertaining whether SC patients made significantly more intrafigural pattern errors than healthy controls when recalling symmetrical configurations.

\section{Method}

\subsection{Participants}

Twenty SC inpatients ( 10 females) and 20 control, healthy adults ( 9 females) participated in the study. The two groups were matched for chronological age $[M($ schizophrenics $)=35.30$ years $(S D=14.57)$ vs. $M($ controls $)=35.20$ years $(S D=12.07) ; t(38)=-0.024, p=0.98]$, but there was a marginal trend for control adults to report more years of formal education $[M($ controls $)=15.0$ vs. $M$ (schizophrenics $)=$ 13.2 years, $t(31.36)=-1.76, p=0.088$ ]. SC patients were recruited from the Acute Psychiatric Care Unit of the Hospital 'San Giovanni Evangelista' (Tivoli, Rome), after approval of the local Research Ethics Board. No participant had a history of traumatic brain injury, epilepsy, substance abuse or other neuropsychological disorders. The diagnosis of schizophrenia was based on the DSM-IV criteria (American Psychiatric Association, 1994), as determined by the joint consensus of the senior psychiatrists of the research team. All patients were stabilized at the time of testing and treated with antipsychotic drugs (13 with firstgeneration antipsychotics and 9 with second-generation antipsychotics). The mean duration of illness was 6.5 years. Symptom severity indexes, as assessed with the Positive and Negative Syndrome Scale 280 for Schizophrenia (PANSS: Kay, Fiszbein, \& Opler, 1987), were respec- 281 tively 16.9 (positive symptoms), 20.6 (negative symptoms) and 32.8282 (general psychopathology).

\subsection{Materials and procedure}

Stimuli were 96 patterns containing 25 squares $(2 \mathrm{~cm} \times 2 \mathrm{~cm}$ each $), 285$ arranged in a $5 \times 5$ matrix (Kemps, 2001; Rossi-Arnaud et al., 2006, 286 2012; see Fig. 1). They were divided in four sub-sets of 24 symmetrical 287 (vertical, horizontal and diagonal) and asymmetrical configurations. 288 Within each sub-set, there were three different patterns for all length 289 levels between 2 and 9 squares. The to-be-recalled squares appeared 290 in red, while all other squares remained black. Presentation was simul- Q4 taneous at encoding - that is, the whole pattern of red and black 292 squares appeared at the same time on the screen and remained visible 293 for a fixed presentation time (see below).

Testing was controlled on a laptop with a 15.4-inch screen and a res- 295 olution of $1280 \times 800$ pixels. During the study phase participants were 296 instructed to remember the locations of the red squares. Each trial 297 began with a fixation point for $500 \mathrm{~ms}$, followed by a target configura- 298 tion $(16 \times 16 \mathrm{~cm})$ for $3000 \mathrm{~ms}$. After a pause of $2000 \mathrm{~ms}$ (during which 299 the screen remained blank), three question marks signalled the begin- 300 ning of the retrieval phase. Participants were required to recall the posi- 301 tions using an appropriate booklet containing 24 blank matrices of the 302 same size as those reproduced on the computer (Andrade, Kemps, 303 Werniers, May, \& Szmalec, 2002). Each matrix was printed on a full 304 page and participants provided their responses by marking the studied 305 squares. For both symmetrical and asymmetrical stimuli, testing started 306 with the presentation of 2-square patterns and continued in ascending 307 order of difficulty until 9-square patterns. The order of vertical, diagonal, 308 horizontal and asymmetrical conditions was counterbalanced across 309 participants. Viewing distance was about $50 \mathrm{~cm}$.

Following the framework proposed by Lecerf and de Ribaupierre 311 (2005), memory errors of SC patients and healthy controls in the recall 312

\section{Procedure}

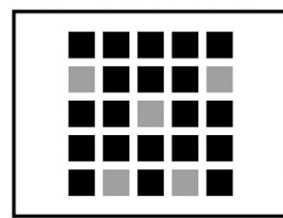

Encoding (3 s)

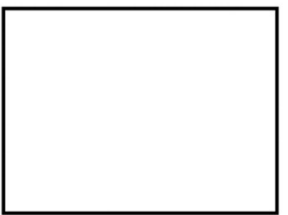

Pause (2 s)

Type of configuration

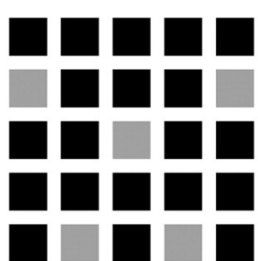

Vertical

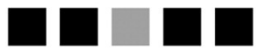

口n日a

.

.

Diagonal

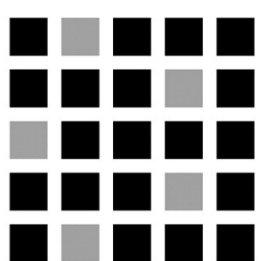

Horizontal

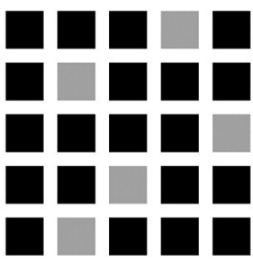

Asymmetric

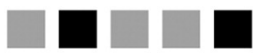

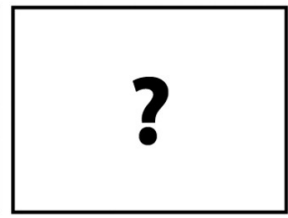

Recall
Fig. 1. Materials and procedure employed in the present experiment. 
of symmetrical patterns were classified in three categories. Extrafigural errors occurred when the entire configuration, or part of it, was reproduced in a different position of the matrix, but both the symmetrical organization of the whole pattern and the relationships between the individual positions were preserved, thus suggesting a specific deficit in the recall of extrafigural information (i.e., information about the localization of the visuospatial pattern with respect to an external frame of reference). One such error is illustrated in Fig. 2 (Extrafigural error). In that case, the SC patient (AL, 31 years) correctly recalled that the two bottom squares were presented in symmetrical positions on the outermost columns, but erroneously located them in the last row of the matrix. Intrafigural path errors occurred when the symmetrical structure of the overall pattern was maintained, but the relationships between individual squares were altered. For instance, in the example reported in Fig. 2 (Intrafigural path error), the patient VA (36 years) incorrectly reproduced a couple of horizontally symmetrical squares as if they

\section{Extrafigural error}
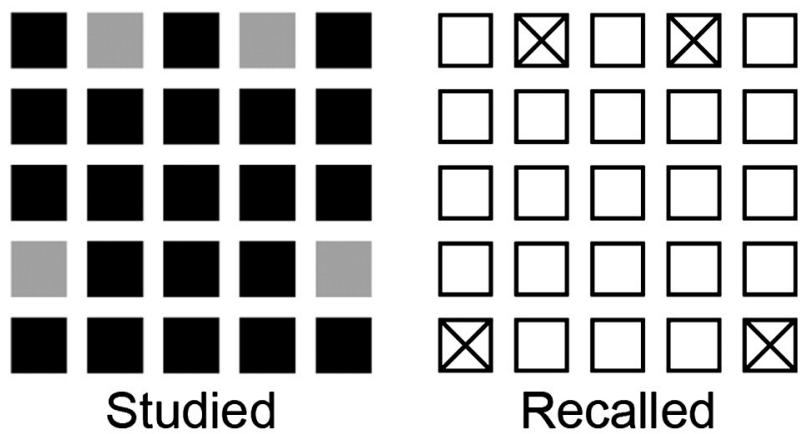

\section{Intrafigural path error}
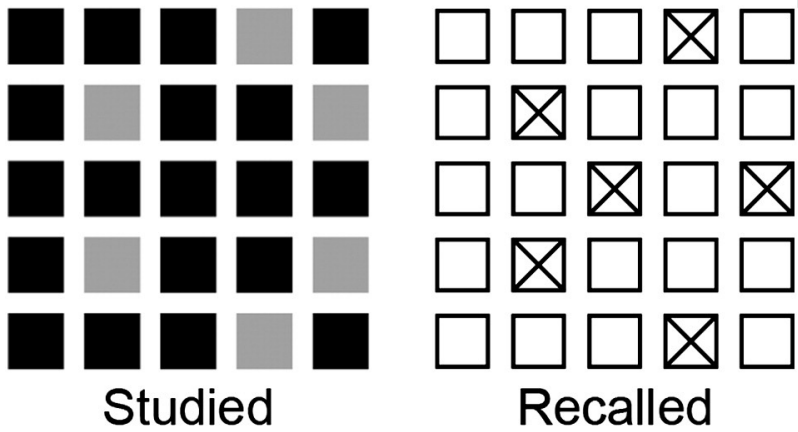

Recalled

\section{Intrafigural pattern error}
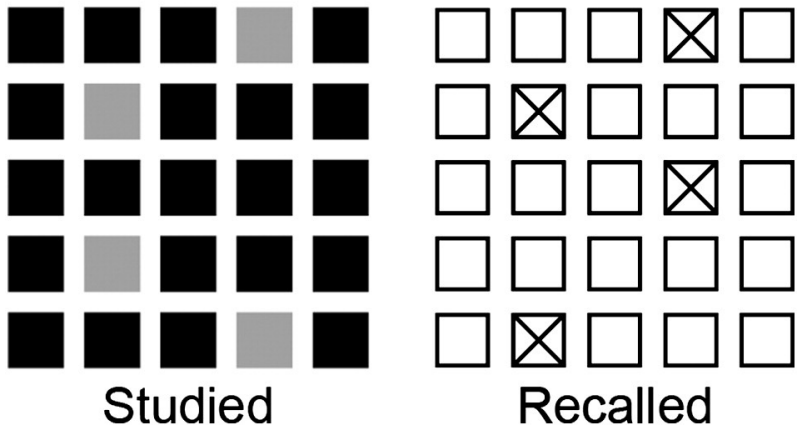

were placed along the central (third) row of the matrix. These errors 329 suggest a specific impairment in the recall of intrafigural path informa- 330 tion (i.e., information about the way in which individual positions are 331 related to each other). Finally, intrafigural pattern errors occurred 332 when the reproduced patterns were no longer symmetrical (see 333 Intrafigural pattern error of Fig. 2), thus demonstrating a deficit in the 334 recall of information about the global visual structure of symmetrical 335 patterns. Indeed, as mentioned above, the advantage offered by sym- 336 metrical stimuli is that individual positions can be bound together to 337 form global visual configurations (Jiang, Olson, \& Chun, 2000; Karlsen 338 et al., 2010; Rossi-Arnaud et al., 2012).

\section{Results}

Fig. 3 illustrates the mean proportion of squares correctly recalled by 342 SC patients and healthy controls, for vertical, horizontal diagonal and 343 asymmetrical patterns, collapsed across all length levels. Data were 344 analysed through a mixed 4 (Pattern Type: vertical, horizontal, diagonal, 345 asymmetrical) $\times 2$ (Group: schizophrenics vs. controls) ANCOVA, consid- 346 ering Pattern Type as the within-subjects factor, Group as the between- 347 subjects factor and Education as the covariate. Results showed significant 348 main effects of both Pattern Type $[F(3,111)=3.81$, MSE $=0.003, p<0.05,349$ $\left.\eta^{2}=0.09\right]$ and Group $\left[F(1,37)=20.65\right.$, MSE $\left.=0.019, p<0.001, \eta^{2}=0.36\right], 350$ indicating that: (i) performance was higher for controls than for SC pa- 351 tients ( $M=0.84$ vs. $M=0.74$ ); (ii) performance decreased linearly 352 from vertical to asymmetrical patterns, with all pairwise comparisons 353 being significant (all $p s<0.01$ ). These effects were qualified by a signifi- 354 cant two-way interaction between Pattern Type and Group $[F(3,355$ $111)=9.12$, MSE $=0.003, p<0.001, \eta^{2}=0.20$ ]. A follow-up analysis of 356 simple effects revealed that the differences between controls and SC pa- 357 tients were significant for horizontal, diagonal and asymmetrical patterns 358 $\left[F(1,37)=29.55, \mathrm{MSE}=0.007, p<0.001, \eta^{2}=0.44 ; F(1,37)=7.56,359\right.$ MSE $=0.006, p<0.01, \eta^{2}=0.17 ; F(1,37)=22.34$, MSE $=0.008,360$ $\left.p<0.001, \eta^{2}=0.38\right]$, and marginally significant for vertical patterns 361 $\left[F(1,37)=3.75, \mathrm{MSE}=0.005, p=0.06, \eta^{2}=0.09\right]$. Importantly, the 362 same analysis indicated that SC patients recalled vertical, horizontal 363 and diagonal patterns better than asymmetrical arrays ( $p<0.001$ for all 364 comparisons), confirming that SC patients are able to use long-term 365 knowledge about symmetry to enhance their memory performance 366 (Baddeley, 2007; Imbo et al., 2009; Kemps, 2001; Knight et al., 2000; 367 Silverstein et al., 1998, 2005). There were, however, small discrepancies 368 between the two groups: in particular, healthy controls recalled vertical 369 and horizontal patterns equally well $(p=0.99)$, whereas SC patients 370

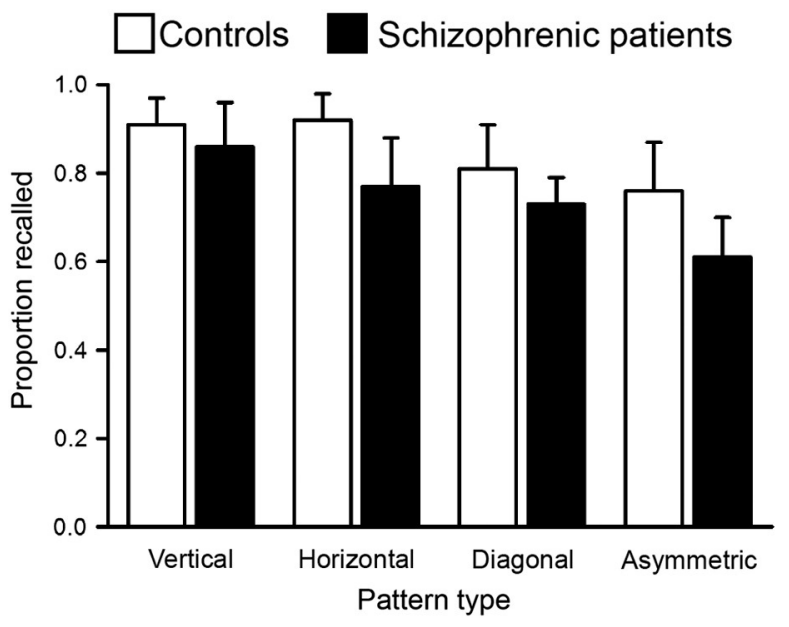


showed a significant advantage for vertical patterns $(p<0.001)$; conversely, SC patients recalled horizontal and diagonal patterns with the same accuracy $(p=0.46)$, whereas healthy controls had a better performance for horizontal than for diagonal patterns $(p<0.001)$. The latter contrasts suggest that the saliency of horizontal symmetry may be reduced in SC patients.

As discussed above, the examination of the overall performance does not allow detailed conclusions about the nature of the processes impaired in SC, for two reasons: a) because accuracy is collapsed across all length levels; and b) because the recall of symmetrical and asymmetrical patterns relies to a different extent on spatial (extrafigural and intrafigural path) and visual (intrafigural pattern) processes. These two questions will be tackled in paragraphs 3.2 and 3.3, respectively.

\subsection{Effects of length level}

To ascertain whether schizophrenic patients showed a deficit when confronted with supercapacity arrays and whether this impairment was greater than that observed with healthy controls, data were analysed through a series of mixed 3 (Length Level: $2-4,5-7,8-9$ squares) $\times 2$ (Group: schizophrenics vs. controls) ANCOVAs, one for each Pattern Type (vertical, horizontal, diagonal and asymmetrical patterns). Education was again included in the models as a covariate. The choice of length levels was primarily intended to ensure the possibility to evaluate the performance of SC patients at both sub- and super-capacity levels (Gold et al., 2003). Based on the span scores obtained in previous studies with similar patterns and a simultaneous presentation (Pieroni et al., 2011; Rossi-Arnaud et al., 2012), we reasoned that: a) set size
2-4 assessed recall performance at sub-capacity levels for all types of 397 patterns (including asymmetrical ones); b) set size 5-7 assessed recall 398 performance at super-capacity levels for asymmetrical and diagonal 399 patterns; and c) set size 8-9 assessed recall performance at super- 400 capacity levels for horizontal and vertical patterns.

401

For vertical symmetry (Fig. 4, left upper panel), results showed sig- 402 nificant main effects of both Length Level $[F(2,74)=12.14$, MSE $=403$ $\left.0.003, p<0.001, \eta^{2}=0.25\right]$ and Group $[F(1,37)=6.230$, MSE $=0.011,404$ $p<0.05, \eta^{2}=0.15$, which were qualified by a significant two-way inter- 405 action $\left[F(2,74)=3.97\right.$, MSE $\left.=0.003, p<0.05, \eta^{2}=0.10\right]$. A follow-up 406 analysis of simple effects, using the Bonferroni adjustment, demonstrat- 407 ed that controls outperformed SC patients when tested with 8-9 square 408 patterns $\left[F(1,37)=10.30, \operatorname{MSE}=0.008, p<0.01, \eta^{2}=0.22\right]$, but not with 409 $2-4$ and $5-7$ square patterns $[F(1,37)<1.15, p>0.29]$.

For horizontal (Fig. 4, right upper panel) and diagonal symmetry 411 (Fig. 4, left bottom panel), the main effects of Length Level and Group 412 were again significant [for horizontal patterns: $F(2,74)=12.01,413$ MSE $=0.005, p<0.001, \eta^{2}=0.25$, and $F(1,37)=29.63$, MSE $=0.016,414$ $p<0.001, \eta^{2}=0.44$; for diagonal patterns: $F(2,74)=13.99$, MSE $=415$ $0.004, p<0.001, \eta^{2}=0.27$, and $F(1,37)=8.59$, MSE $=0.015, p<0.01,416$ $\eta^{2}=0.19$ ], as they were the interactions between the two factors [for 417 horizontal patterns: $F(2,74)=15.64$, MSE $=0.005, p<0.001, \eta^{2}=0.30 ; 418$ for diagonal patterns: $F(2,74)=4.14$, MSE $\left.=0.004, p<0.05, \eta^{2}=0.10\right] .419$ Follow-up analyses of simple effects indicated that, with both types of 420 patterns, healthy controls outperformed SC patients at medium(5-7 421 squares) and high length levels (8-9 squares) [for horizontal patterns: 422 $F(1,37)=27.34$, MSE $=0.013, p<0.001, \eta^{2}=0.42$, and $F(1,37)=423$ 28.39, MSE $=0.010, p<0.001, \eta^{2}=0.43$; for diagonal patterns: $F(1,424$
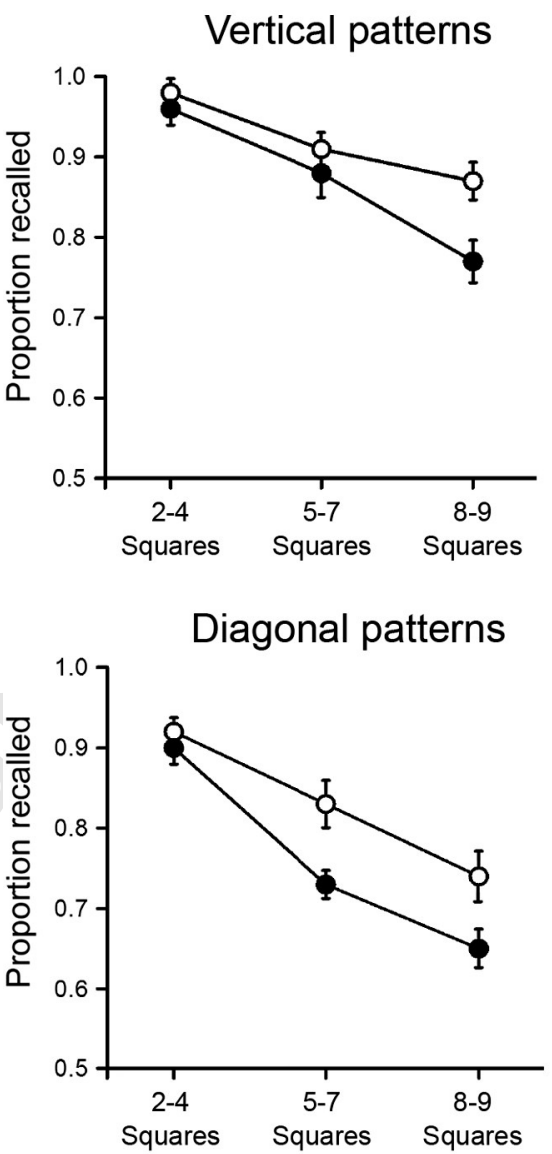
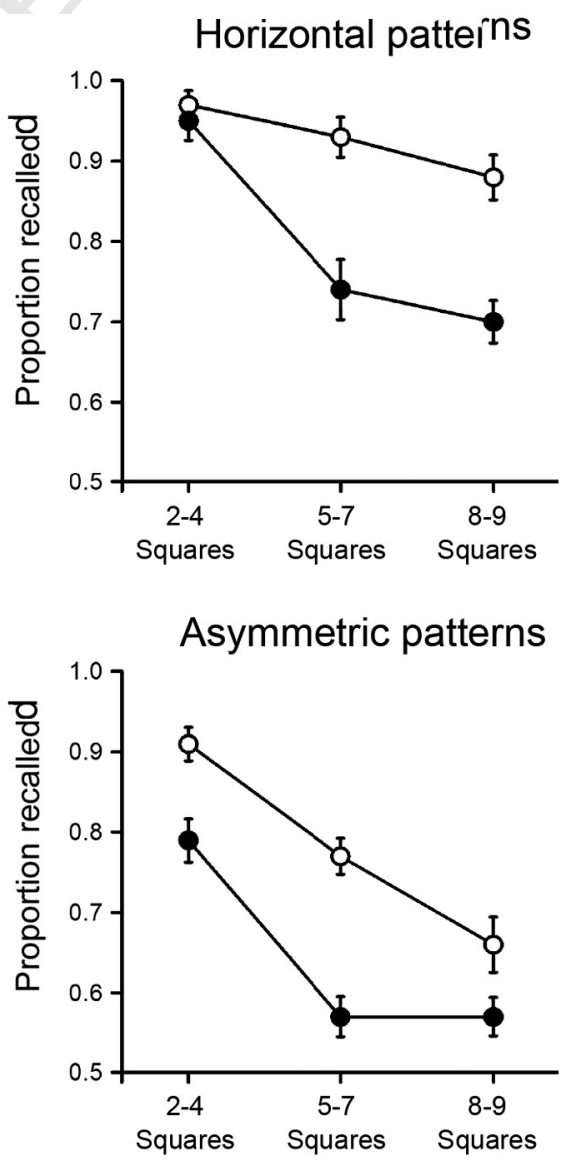

\section{Controls Schizophrenic patients}

Fig. 4. Proportions of squares correctly recalled, as a function of Group, Pattern Type and Length Level. 
$37)=12.34$, MSE $=0.007, p \leq 0.001, \eta^{2}=0.25$, and $F(1,37)=5.62$, MSE $=0.013, p<0.05, \eta^{2}=0.13$, but not at low length levels $(2-4$ squares) [for horizontal patterns: $F(1,37)=2.80, p=0.10$; for diagonal patterns: $F(1,37)=1.18, p=0.28$.

Finally, a significant interaction between Length Level and Group was also obtained in the analysis concerning asymmetrical patterns $\left[F(2,74)=5.07, \mathrm{MSE}=0.006, p<0.01, \eta^{2}=0.12\right]$. However, in the latter case the difference between SC patients and healthy controls was significant in all conditions (Fig. 4, right bottom panel) [for 2-4 square patterns: $F(1,37)=17.76$, MSE $=0.006, p<0.001, \eta^{2}=0.32$; for $5-7$ square patterns: $F(1,37)=36.89$, MSE $=0.010, p<0.001, \eta^{2}=0.50$; for 8-9 square patterns: $F(1,37)=4.24$, MSE $=0.018, p<0.05, \eta^{2}=$ $0.10]^{1}$.

One problem with the above analyses is that the recall accuracy for 2 to 4 square vertical, horizontal and diagonal patterns, and for 5 to 7 square vertical patterns was near ceiling, raising questions about the extent to which these data violate the typical assumptions of parametric analyses (e.g., normality, equality of variance). Regarding vertical patterns, it is worth noting that the performance of both controls and SC patients increased from medium to high length levels $[t(19)=4.18$, $\mathrm{p}<0.01$ and $t(19)=4.56, \mathrm{p}<0.001$ ]: thus, the absence of significant differences between the two groups with 5-7 square patterns cannot be simply explained by a ceiling effect. Nonetheless, non-parametric analyses were conducted to verify the foregoing results. A series of MannWhitney $U$-tests confirmed that the recall accuracy of SC patients and healthy controls did not differ for 2-4 and 5-7 square vertical patterns [Zs $>-1.36, p>0.18$ ], and for $2-4$ square horizontal and diagonal patterns $[Z>-1.18, p>0.29]$. In contrast, the difference between the two groups was significant for $2-4$ square asymmetrical patterns $[Z=-4.08, p<0.001]$.

\subsection{Hierarchical regression analyses}

One purpose of the present study was to ascertain whether SC patients showed a significant deficit in the retrieval of visual global images of the studied patterns, after removing variance associated with the use of extrafigural and intrafigural path processes. To examine this issue, we used the method illustrated by Lorsbach and Reimer (2005). Using hierarchical multiple regression analyses, these authors found that age remained a significant predictor of memory performance in the combination condition of an object-location task, even when the variance associated with children's performance in the single-feature conditions was removed. Here, we applied the same procedure to verify whether the differences between SC patients and healthy controls in the recall of symmetrical patterns (i.e., in the use of intrafigural pattern processes) remained significant after removing variance associated with performance on asymmetrical patterns (i.e., with the use of extrafigural and intrafigural path processes). The rationale was provided by previous evidence suggesting that, with a simultaneous presentation, the recall of asymmetrical patterns is primarily based on spatial extrafigural and intrafigural path processes, whereas the recall of symmetrical patterns requires a greater involvement of visual intrafigural pattern processes (Lecerf \& de Ribaupierre, 2005; Rossi-Arnaud et al., 2012). Following Lorsbach and Reimer (2005), in the first step the recall accuracy with asymmetrical patterns (i.e., the variable whose contribution had to be partialled out) was entered in the regression model as the initial predictor. Then, in the second step, we added to the equation the main effect of group, in the form of a contrast dummy variable (by assigning the values 0 and 1 to SC patients and healthy controls, respectively; Frazier, Tix, \& Barron, 2004). The dependent measure was the participants' performance with symmetrical patterns. We only focused on those length levels in which differences between SC patients and healthy controls were significant (i.e., 8-9 square patterns for vertical symmetry and 5-7 and 8-9 square patterns for horizontal and diagonal symmetry).
Table 1

Hierarchical regression analyses.

Cl.2

\begin{tabular}{|c|c|c|c|c|c|c|}
\hline & $\beta$ & $t$ & $\Delta R$ & $R^{2}\left(\operatorname{Adj} R^{2}\right)$ & \multirow{2}{*}{$\begin{array}{l}\mathrm{t} 1.3 \\
\mathrm{t} 1.4\end{array}$} \\
\hline & & \multicolumn{4}{|c|}{$\begin{array}{l}\text { Vertical symmetry } \\
\text { (8-9 square patterns) }\end{array}$} & \\
\hline Step 1 & $\begin{array}{l}\text { Accuracy asymmetrical patterns } \\
\text { (8-9 squares) }\end{array}$ & 0.60 & $5.31^{* * *}$ & 0.521 & $0.521(0.508)$ & $\mathrm{t} 1.5$ \\
\hline \multirow[t]{2}{*}{ Step 2} & Group difference & 0.31 & $2.74^{* *}$ & 0.081 & $0.601(0.580)$ & $\begin{array}{l}\mathrm{t} 1.6 \\
\mathrm{t} 1.7\end{array}$ \\
\hline & & \multicolumn{4}{|c|}{$\begin{array}{l}\text { Horizontal symmetry } \\
\text { (8-9 square patterns) }\end{array}$} & $\mathrm{t} 1.8$ \\
\hline Step 1 & $\begin{array}{l}\text { Accuracy asymmetrical patterns } \\
\text { (8-9 squares) }\end{array}$ & 0.40 & $3.69^{* * *}$ & 0.380 & $0.380(0.363)$ & $\mathrm{t} 1.9$ \\
\hline \multirow[t]{2}{*}{ Step 2} & Group difference & 0.54 & $4.96^{* * *}$ & 0.248 & $0.628(0.607)$ & $\mathrm{t} 1.10$ \\
\hline & & \multicolumn{4}{|c|}{$\begin{array}{l}\text { Horizontal symmetry } \\
\text { (5-7 square patterns) }\end{array}$} & $\mathrm{t} 1.12$ \\
\hline Step 1 & $\begin{array}{l}\text { Accuracy asymmetrical patterns } \\
\text { (5-7 squares) }\end{array}$ & 0.53 & $3.48^{* * *}$ & 0.570 & $0.570(0.559)$ & $\mathrm{t} 1.13$ \\
\hline \multirow[t]{2}{*}{ Step 2} & Group difference & 0.30 & $1.96^{*}$ & 0.040 & $0.611(0.590)$ & $\mathrm{t} 1.14$ \\
\hline & & \multicolumn{4}{|c|}{$\begin{array}{l}\text { Diagonal symmetry } \\
\text { (8-9 square patterns) }\end{array}$} & $\mathrm{t} 1.16$ \\
\hline Step 1 & $\begin{array}{l}\text { Accuracy asymmetrical patterns } \\
\text { (8-9 squares) }\end{array}$ & 0.63 & $5.18^{* * *}$ & 0.503 & $0.503(0.490)$ & $\mathrm{t} 1.17$ \\
\hline \multirow[t]{2}{*}{ Step 2} & Group difference & 0.20 & 1.64 & 0.034 & $0.537(0.512)$ & $\mathrm{t} 1.18$ \\
\hline & & \multicolumn{4}{|c|}{$\begin{array}{l}\text { Diagonal symmetry } \\
\text { (5-7 square patterns) }\end{array}$} & $\mathrm{t} 1.20$ \\
\hline Step 1 & $\begin{array}{l}\text { Accuracy asymmetrical patterns } \\
\text { (5-7 squares) }\end{array}$ & 0.62 & $3.61^{* * *}$ & 0.492 & $0.492(0.479)$ & $\mathrm{t} 1.21$ \\
\hline Step 2 & Group difference & 0.09 & 0.57 & 0.004 & $0.497(0.469)$ & $\mathrm{t} 1.22$ \\
\hline & & & & & & $\mathrm{t} 1.23$ \\
\hline & & & & & & $\mathrm{t} 1.24$ \\
\hline
\end{tabular}

The results of these multiple regression analyses are illustrated in 488 Table 1 . As can be noted, the performance with symmetrical patterns 489 could be always predicted from the recall accuracy with asymmetrical 490 patterns [for 8-9 square vertical symmetry: $F(1,38)=41.26$, MSE $=491$ $0.007, p<0.001$; for $8-9$ and 5-7 square horizontal symmetry: $F(1,492$ $38)=23.25$, MSE $=0.012, p<0.001$, and $F(1,38)=50.41$, MSE $=493$ $0.010, p<0.001$; for $8-9$ and $5-7$ square diagonal symmetry: $F(1,494$ $38)=38.50$, MSE $=0.009, p<0.001$, and $F(1,38)=36.82$, MSE $=495$ $0.006, p<0.001]$. In addition, group membership significantly improved 496 the prediction of the dependent variable for vertical and horizontal 497 symmetry [for 8-9 square vertical symmetry: $F(1,37)=7.49,498$ $p<0.01$; for 8-9 and 5-7 square horizontal symmetry: $F(1,37)=499$ $24.62, p<0.001$, and $F(1,38)=3.84, p=0.05$ ], but not for diagonal sym- 500 metry $[F(1,37)=2.68, p=0.11$, and $F(1,37)=0.32, p=0.57]$. 501

In sum, these regression analyses indicated that, with supra-span ver- 502 tical and horizontal patterns, SC patients showed a significant impairment 503 in the retrieval of visual global images of the studied patterns, even after 504 accounting for variance associated with the use of extrafigural and 505 intrafigural path processes. The null result with diagonal stimuli is consis- 506 tent with previous evidence showing that this type of symmetry is the 507 most difficult to be detected in visual discrimination tasks (Wenderoth, 508 1994) and the latest to develop in young children (Bornstein \& Stiles- 509 Davis, 1984). Therefore, the role of intrafigural pattern processes may 510 have been quite limited for diagonal configurations, explaining the lack 511 of significant differences between SC patients and healthy controls. 512

\subsection{Error analysis}

Error frequencies for symmetrical patterns were analysed through 514 a series of mixed 3 (Error Type: extrafigural errors, intrafigural path er- 515 rors and intrafigural pattern errors) $\times 3$ (Length Level: 2-4, 5-7, 8-9 516 squares) $\times 2$ (Group: schizophrenics vs. controls) ANCOVAs, with 517 
Error Type and Length Level as repeated factors, Group as the betweensubjects factor and Education as the covariate. For the purpose of statistical analyses, the raw frequencies of the three types of errors were transformed into proportions, by dividing by the total number of patterns presented at each length level (i.e., for the maximum number of possible errors).

For vertical patterns (Fig. 5, upper panel), the results showed significant main effects of Length Level $[F(2,74)=22.63$, MSE $=0.006$ $\left.p<0.001, \eta^{2}=0.38\right]$ and Group $[F(1,37)=7.31$, MSE $=0.017$, $p \leq 0.01, \eta^{2}=0.17$ ], indicating that: (i) error rates were lower for $2-4$ than for 5-7 square patterns $(M=0.01$ vs. $M=0.08, p<0.001)$, and 528 lower for 5-7 than for 8-9 square patterns ( $M=0.08$ vs. $M=0.15,529$ $p<0.001$ ); (ii) SC patients made more errors than healthy controls 530 ( $M=0.10$ vs. $M=0.06$ ). The main effect of Error Type did not reach 531 the significance level $[F(2,74)=1.30, p=0.27]$, but the two-way inter- 532 actions between Error Type and Group and between Error Type and 533 Length Level were significant $[F(2,74)=4.57$, MSE $=0.027, p \leq 0.01,534$ $\eta^{2}=0.11$ and $F(4,148)=2.74$, MSE $=0.016, p<0.05, \eta^{2}=0.07$, respec- 535 tively], as well as the three-way interaction between all factors $[F(4,536$ $148)=4.78, \operatorname{MSE}=0.016, p<0.001, \eta^{2}=0.12$ ]. A follow-up analysis 537

Vertical symmetry
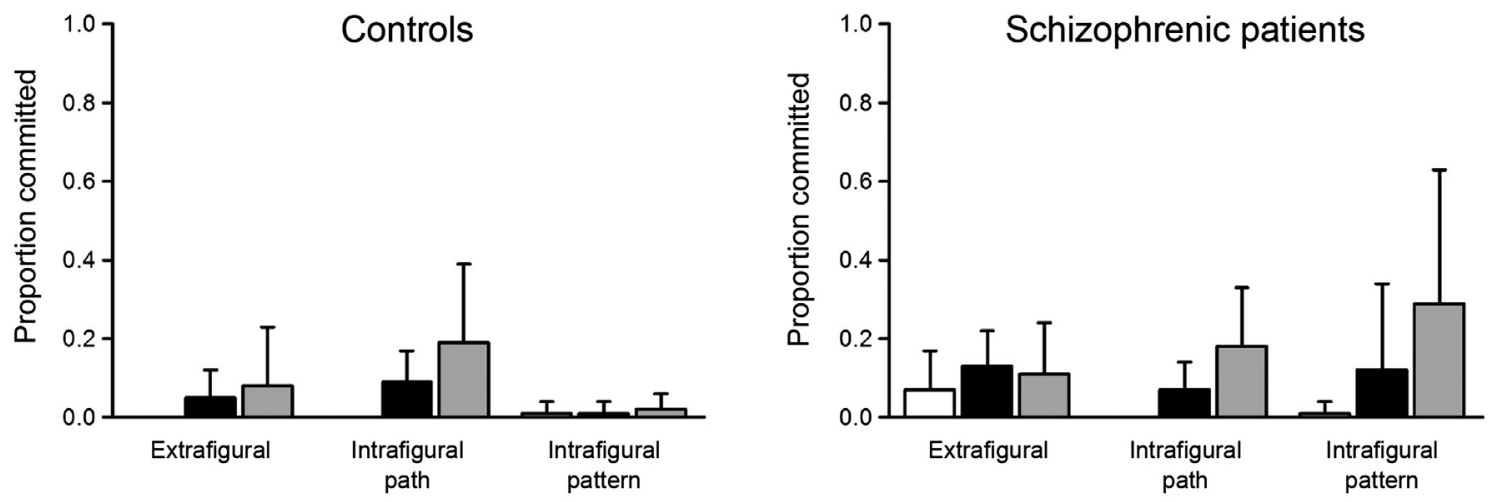

Horizontal symmetry
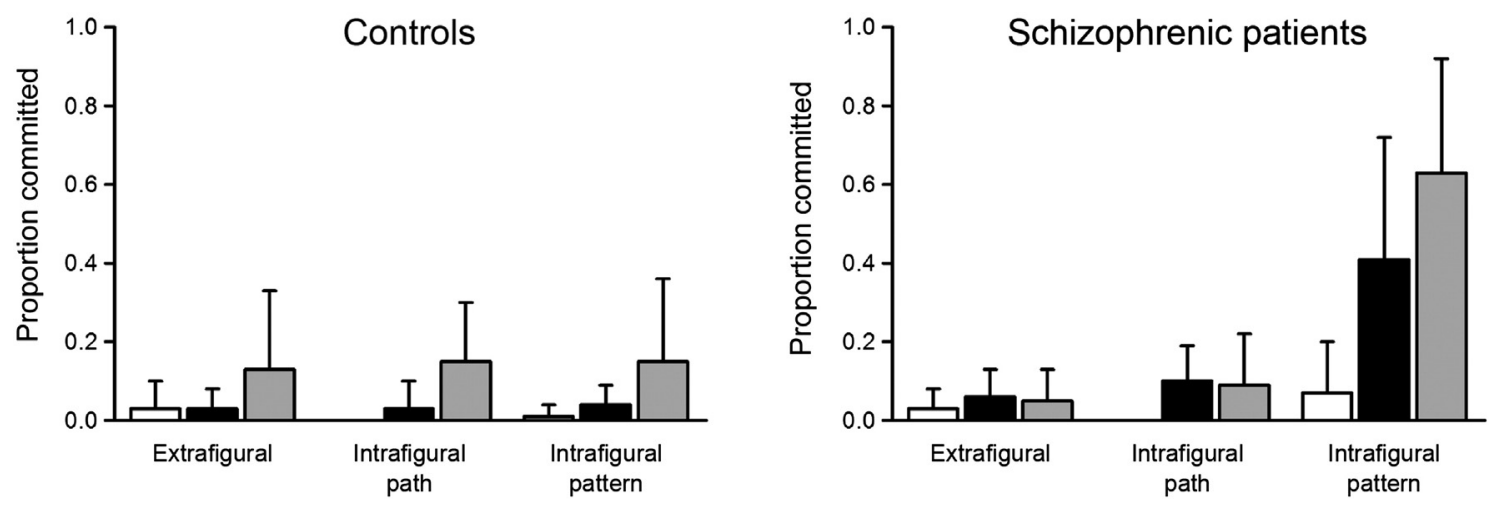

Diagonal symmetry
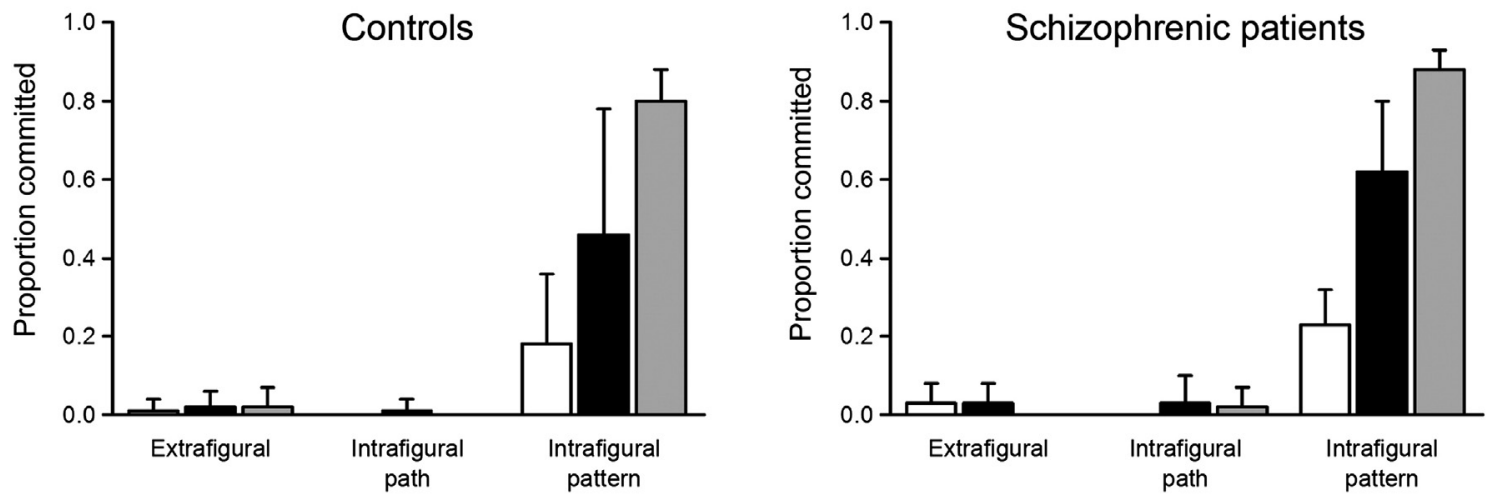

Length 2-4 $\square$ Length 5-7 $\square$ Length 8-9

Fig. 5. Proportions of errors, as a function of Group, Error Type and Pattern Type. 
on the latter interaction revealed that SC patients made significantly more extrafigural errors at low (2-4 squares) and medium (5-7 squares) length levels $\left[F(1,37)=6.69\right.$, MSE $=0.004, p \leq 0.01, \eta^{2}=$ 0.15 and $F(1,37)=5.20$, MSE $=0.008, p<0.05, \eta^{2}=0.12$ ], and more intrafigural pattern errors with 8-9 square patterns $[F(1,37)=9.61$, MSE $\left.=0.055, p<0.01, \eta^{2}=0.21\right]$, compared to healthy controls.

For horizontal patterns (Fig. 5, central panel), the same analysis as above revealed significant main effects for all factors [Error Type: $F(2$, $74)=14.13$, MSE $=0.030, p<0.001, \eta^{2}=0.27$; Length Level: $F(2$, $74)=15.65, \mathrm{MSE}=0.008, p<0.001, \eta^{2}=0.29$; Group: $F(1,37)=$ $34.44, \mathrm{MSE}=0.021, p<0.001, \eta^{2}=0.48$ ], indicating that: (i) intrafigural pattern errors $(M=0.24)$ occurred more frequently than extrafigural and intrafigural path errors $(M=0.05$ and $M=0.06$ ); (ii) error rates were lower with $2-4$ than with $5-7$ square patterns $(M=0.02$ vs. $M=0.12, p<0.001$ ), and lower with 5-7 than with 8-9 square patterns $(M=0.12$ vs. $M=0.21, p<0.001)$; (iii) error rates were higher for SC patients than for healthy controls $(M=0.16$ vs. $M=0.07)$. In addition, there were significant two-way interactions between Error Type and Group $\left[F(2,74)=26.72, \mathrm{MSE}=0.030, p<0.001, \eta^{2}=0.41\right]$ and between Length Level and Group $\left[F(2,74)=14.89\right.$, MSE $=0.008, p<0.001, \eta^{2}=$ $0.28]$, which were qualified by a three-way interaction between all variables $\left[F(4,148)=14.13\right.$, MSE $\left.=0.015, p<0.001, \eta^{2}=0.27\right]$. A follow-up analysis on the latter interaction showed that the differences between SC patients and healthy controls were significant for intrafigural path errors at medium length levels ( $5-7$ squares) $[F(1,37)=8.44$, MSE $=$ $0.007, p<0.01, \eta^{2}=0.19$ ] and for intrafigural pattern errors at medium and high length levels ( $5-7$ and $8-9$ squares) $[F(1,37)=24.72$, MSE $=$ $0.040, p<0.001, \eta^{2}=0.40$ and $F(1,37)=46.91$, MSE $=0.047, p<0.001$, $\left.\eta^{2}=0.56\right]$

Finally, for diagonal patterns (Fig. 5, bottom panel), the ANOVA found significant main effects for all factors [Error Type: $F(2,74)=$ 50.62, MSE $=0.026, p<0.001, \eta^{2}=0.57$; Length Level: $F(2,74)=$ 19.81, MSE $=0.006, p<0.001, \eta^{2}=0.34$; Group: $F(1,37)=4.51$, MSE $\left.=0.026, p<0.05, \eta^{2}=0.10\right]$, together with a marginally significant interaction between Error Type and Group $[F(2,74)=3.01$, MSE $=$ $\left.0.026, p=0.055, \eta^{2}=0.07\right]$. A follow-up analysis indicated that, compared to healthy controls, SC patients made more intrafigural pattern errors at high length levels (8-9 squares) $[F(1,37)=3.60$, MSE $=$ $\left.0.025, p=0.066, \eta^{2}=0.09\right]$, whereas there were no differences between the two groups with respect to extrafigural and intrafigural path errors $[F(1,37)<1.21, p>0.27]$.

\section{Discussion}

The present study aimed at examining the functioning of perceptual binding processes in SC patients and matched healthy controls, by analysing their performance in a WM visuospatial task requiring the recall of symmetrical and asymmetrical patterns varying in length between 2 and 9 items (Rossi-Arnaud et al., 2006, 2012). The results showed that: a) the overall recall accuracy was lower for SC patients than for healthy controls with all types of patterns, but both groups showed the typical advantage of symmetrical (vertical, horizontal and diagonal) over asymmetrical stimuli; b) increasing length levels had larger detrimental effects on SC patients than on controls, with both symmetrical and asymmetrical configurations; c) the impairment of SC patients in the recall of 8-9 square vertical patterns and 5-7 and 8-9 square horizontal patterns remained significant after removing the variance associated with performance on asymmetrical patterns; and d) SC patients made significantly more intrafigural pattern errors (i.e., errors in which the symmetrical organization of the studied patterns were lost) when recalling 8-9 square vertical configurations, and 5-7 and 8-9 square horizontal and diagonal configurations. The latter two findings suggest a selective deficit in the ability to form and retrieve the visual global images of supra-span symmetrical configurations.

Regarding our first aim, the analysis of the overall performance showed that both SC patients and healthy controls recalled symmetrical patterns significantly better than asymmetrical ones. These results con- 602 firm earlier evidence indicating that SC patients are not impaired in the 603 processing of stimuli with strong configurational properties (Knight 604 et al., 2000; Uhlhaas \& Silverstein, 2005) and that they are able to ex- 605 ploit the symmetrical structure of the to-be-remembered patterns in 606 order to improve their memory span (Baddeley, 2007; Imbo et al., 607 2009; Kemps, 2001; Silverstein et al., 1998, 2005). In agreement, 608 Knight et al. (2000) examined the performance of SC patients, de- 609 pressed individuals and healthy controls in a same-different judgement 610 task in which they had to decide whether symmetric letter pairs were 611 physically the same (i.e., the same letter in the same orientation). In 612 this condition, the typical result is that decision times are slower to sym- 613 metrical (e.g., vertical-axis bilateral) than to asymmetrical letter pairs. 614 Importantly, such symmetry interference was obtained in all groups, 615 suggesting that SC patients processed symmetrical stimuli as gestalts 616 that had to be broken down so that element comparison could proceed 617 (Knight et al., 2000). More pertinent for the present study, Silverstein 618 et al. (2005) showed that both SC patients and healthy controls 619 recognised symmetrical arrays of six asterisks better than asymmetrical 620 arrays, although the accuracy of SC patients was significantly worse 621 than that of controls; furthermore, the patients' performance with reg- 622 ular patterns normally increased as a function of repeated exposure, 623 whereas memory for asymmetric stimuli did not improve, indicating 624 that SC patients were less able to consolidate novel, unstructured visual 625 information (relative to controls).

However, unlike previous studies, we parametrically manipulated the 627 length of the to-be-remembered patterns, introduced a detailed classifi- 628 cation of error types and evaluated the independent contributions of spa- 629 tial (extrafigural and intrafigural path) and visual (intrafigural pattern) 630 processes to recall accuracy. These additional analyses qualified the fore- 631 going general conclusions in important ways. First, when the perfor- 632 mance of the two groups was analysed in terms of increasing length 633 levels, it turned out that SC patients were significantly impaired in the re- 634 call of supercapacity symmetrical arrays (5-7 square patterns for hori- 635 zontal and diagonal symmetry, and 8-9 square patterns for vertical, 636 horizontal and diagonal symmetry). Analogous findings have been re- 637 ported by Gold et al. (2003), who tested visual WM for single features 638 (colour and orientation) and feature combinations in schizophrenia 639 using a change detection task. They showed that, unlike controls, the 640 WM capacity of SC patients declined from set size 4 to set size 6 , and at- 641 tributed this decrement to a deficit of selective attention. From a theoret- 642 ical point of view, our results support the view that binding processes are 643 relatively automatic (i.e., operating at pre-attentive stages, independent- 644 ly from attentional resources) at low load levels, but become more 645 attention-demanding at medium and high load levels (Kochan et al., 646 2011). Using an object-location task, Kochan et al. (2011) have recently 647 reported that, during the retrieval phase, the performance of healthy 648 adults with high load patterns was associated with a stronger deactiva- 649 tion of the default mode network - an interconnected system of cortical 650 regions which is preferentially active when the brain is in a state of wake- 651 ful rest and memory load is low (Raichle \& Snyder, 2007) - and argued 652 that binding becomes a resource-intensive process at higher memory 653 loads. Interestingly, a large number of studies have reported dysfunctions 654 of the default mode network in schizophrenia (including hyperactiva- 655 tion: see Whitfield-Gabrieli \& Ford, 2012, for a review), potentially ac- 656 counting for the deficits exhibited by our SC patients with supra-span 657 patterns. Kochan et al. (2011) further proposed that bound representa- 658 tions may be more fragile than single feature representations (Allen 659 et al., 2006), and that they may be more easily disrupted when demands 660 on visuospatial attention are high at retrieval. Accordingly, in the present 661 study, the need to recall increasing amounts of items (and their spatial 662 relationships) may have led to concurrent impairments in the ability to 663 bind the short-term information of the visuospatial sketchpad with the 664 LTM knowledge about symmetry.

In addition, we used multiple regression analyses to tease apart the 666 contribution of extrafigural and intrafigural spatial processes (responsible 667 
for coding the position of the pattern with respect to an external frame of reference and the spatial relationships between individual items) from that of intrafigural visual processes (responsible for remembering the arrays as integrated, global configurations; Lecerf \& de Ribaupierre, 2005). The results showed that, at medium and high length levels, the differences between SC patients and healthy controls in the recall of vertical and horizontal patterns (which was assumed to require a more extensive use of intrafigural visual processes) remained significant even after removing the variance associated with performance on asymmetrical patterns (which was assumed to reflect primarily the use of extrafigural and intrafigural spatial processes). Therefore, when the size of the tobe-remembered patterns exceeded the capacity of the visuospatial sketchpad (about four items, according to Cowan, 2001), SC patients demonstrated significant deficits in the retrieval of the global visual images of studied patterns, above and beyond their impairment in the retrieval of spatial information about individual items.

This conclusion was further supported by the error analysis. In recent years, an increasing number of studies have pointed out that the mechanisms underlying memory errors must be taken into account when comparing the performance of SC patients and healthy controls (Brébion et al., 2005, 2007; Elvevag et al., 2001; Lee et al., 2008). Accuracy measures, like the proportion of squares correctly recalled, can only provide information about the number of items that participants are able to simultaneously store into visuospatial WM, whereas a finer analysis of error types may provide crucial information about the specific processes that are impaired in SC patients (Elvevag et al., 2001; Lee et al., 2008). Lee et al. (2008), for instance, reported that SC patients had an increased frequency of "false memory" errors (i.e., errors with high confidence ratings) and that these errors were associated with higher activation of the prefrontal regions of both hemispheres during the delay interval, suggesting that they encoded incorrect stimuli and maintained their internal representations until the test phase. The present results showed that, compared to healthy controls, SC patients were more likely to reconstruct symmetrical patterns as if they were asymmetrical. Interestingly, this type of errors occurred most frequently with horizontal and diagonal configurations of medium and high length levels, confirming that the formation and the maintenance of global visual images was more demanding for these patterns than for vertical ones (Pieroni et al., 2011; Rossi-Arnaud et al., 2006, 2012). A similar conclusion has been reached by Rossi-Arnaud et al. (2012) with healthy adults. Using a dual-task methodology, these authors showed that the advantage of vertical over asymmetrical patterns was significant for both high and low performers (on the basis of their mean span scores), and was not eliminated by secondary tasks designed to interfere with WM subsystems. In contrast, in the low performing group, the advantage of horizontal and diagonal patterns was abolished by both visuospatial and executive interferences, indicating that, for low performers, the recall of the global visual configurations of horizontal and diagonal patterns may be more dependent upon controlled processes, relative to vertical patterns.

A possible explanation for our findings is that SC patients are less efficient than controls in the development of an effective top-down control over perceptual processes (Schwartz Place \& Gilmore, 1980; Silverstein et al., 1996). Several studies have suggested that SC patients might have a deficit "in the ability of current sensory input to initiate a simultaneous recreation of aspects of experience associated with past occurrences of the stimulus" (Silverstein et al., 1996, p. 411). Schwartz Place and Gilmore (1980), for instance, found that, in contrast to controls, SC patients did not show superior grouping of stimuli after a repeated exposure to other grouped configurations, suggesting a weaker influence of the regularity of previous input on current perception. Similarly, Knight et al. (2000) reported that a familiarization with vertical stimuli enhanced the ability of controls to use the presence of this type of symmetry as a diagnostic for subsequent responses, whereas SC patients failed to learn this strategy. In the present study, presentation was blocked by symmetry (with all patterns of a given type being tested in sequence), a condition which creates a sustained top-down 734 mental set for a particular kind of processing (Jaswal \& Logie, 2013). 735 The implication is that, with increasing task difficulty and SC patients 736 being progressively overwhelmed by the high number of items to be re- 737 membered, they might have become less able to exploit knowledge on 738 the type of symmetry being tested to guide retrieval processes. $\quad 739$

A related account is that SC patients may be less efficient in the use of 740 redintegration processes, whereby partially decayed traces are complet- 741 ed on the basis of stored knowledge in LTM (Lewandowsky \& Farrell, 742 2000; Schweickert, 1993). The importance of redintegration for visuo- 743 spatial memory has been demonstrated by Kemps (2001), who explored 744 the short-term recall of irregular (random) and regular sequences in 745 healthy participants. She found that a brief training similar to the Hebb 746 procedure, which leads to the creation of LTM representations, increased 747 the recall of random stimuli to the same levels achieved with structured 748 paths. On the basis of these findings, Kemps (2001) proposed that LTM 749 representations about the structure of regular paths are consulted during 750 the process of retrieving block sequences from visuospatial WM, facilitat- 751 ing their reconstruction when the capacity of the visuospatial sketchpad 752 is exceeded (see Imbo et al., 2009, for a similar argument). As mentioned 753 above, Bornstein and Stiles-Davis (1984), found that healthy adults tend 754 to reproduce symmetrical patterns by using a side-side reflection strate- 755 gy (i.e., retrieving one side of the array and then completing the other 756 side by reflection across the symmetry axis). A plausible hypothesis is 757 that, under high load memory conditions (i.e., when recalling patterns 758 of medium and high length levels), SC patients may be less capable to 759 take advantage of these axis-based strategies, compared to healthy 760 controls.

In conclusion, we found that, although SC patients were less accurate 762 than healthy controls in the recall of all types of stimuli, they maintained 763 the typical advantage of symmetrical over asymmetrical patterns 764 (Pieroni et al., 2011; Rossi-Arnaud et al., 2006, 2012), suggesting an in- 765 tact ability to bind LTM semantic knowledge with the short-term infor- 766 mation held in the visuo-spatial sketchpad. On the other hand, SC 767 patients were significantly impaired in the formation and retrieval of 768 the global visual images of symmetrical supercapacity arrays, as indicat- 769 ed by multiple regression analyses and the finding that they were more 770 likely to reproduce symmetrical patterns as asymmetrical, particularly 771 at medium (5-7 squares) and high (8-9 squares) length levels. The lat- 772 ter results are consistent with the hypothesis that schizophrenia may be 773 associated with a specific deficit in the ability to use the on-line knowl- 774 edge of the type of symmetry being tested to guide retrieval processes 775 (Knight et al., 2000; Schwartz Place \& Gilmore, 1980; Silverstein et al., Q9 1996).

Similar results were achieved when analysing all length levels. A se- Q10 ries of mixed 8 (Length Level: $2-9$ squares) $\times 2$ (Group: schizophrenics 779 vs. controls) ANCOVAs found significant two-way interactions between 780 Group and Length Level for all types of patterns [vertical: $F(7,259)=781$ 2.64, MSE $=0.007, p \leq 0.01, \eta^{2}=0.07$; horizontal: $F(7,259)=6.35,782$ MSE $=0.010, p<0.001, \eta^{2}=0.15$; diagonal: $F(7,259)=2.51$ MSE $=783$ $0.008, p<0.05, \eta^{2}=0.06$; asymmetric: $F(7,259)=5.46$, MSE $=0.011,784$ $p<0.001, \eta^{2}=0.13$ ]. Follow-up analyses of simple effects demonstrated 785 that controls outperformed SC patients: a) with 3- and 9-square vertical 786 patterns (all $p s<0.05$ ); b) with 5-, 6-, 7-, 8- and 9-square horizontal pat- 787 terns (all $p s<0.001$ ); c) with 3-, 6-, 7-, 8- and 9-square diagonal patterns 788 (all ps $<0.05)$; d) with 3-, 4-, 5-, 6-, 7- and 9-square asymmetrical pat- 789 terns (all ps<0.05).

\section{Uncited reference} Q11

Barch, 2005

\section{Acknowledgements}

This study was supported by a grant from Sapienza University 794 awarded to C. Rossi-Arnaud (Ateneo 2011 project no. C26A11RESK). 795 


\section{References}

Aleman, A., Hijman, R., Haan, E. F., \& Kahn, R. S. (1999). Memory impairment in schizophrenia: A meta-analysis. American Journal of Psychiatry, 156, 1358-1366.

Allen, R. J., Baddeley, A.D., \& Hitch, G. J. (2006). Is the binding of visual features in working memory resource-demanding? Journal of Experimental Psychology: General, 135, 298-313.

Allen, R. J., Hitch, G. J., \& Baddeley, A.D. (2009). Cross-modal binding and working memory. Visual Cognition, 17, 83-102.

Allen, R. J., Hitch, G. J., Mate, J., \& Baddeley, A.D. (2012). Feature binding and attention in working memory: A resolution of previous contradictory findings. Quarterly Journal of Experimental Psychology, 65, 2369-2383.

American Psychiatric Association (1994). Diagnostic and statistical manual of mental disorders (4th ed.)Washington, D.C.: Author.

Andrade, J., Kemps, E., Werniers, Y., May, J., \& Szmalec, A. (2002). Insensitivity of visual short-term memory to irrelevant visual information. Quarterly Journal of Experimental Psychology, 55, 753-774.

Baddeley, A.D. (2000). The episodic buffer: A new component of working memory? Trends in Cognitive Sciences, 4, 417-423.

Baddeley, A.D. (2007). Working memory, thought, and action. New York, NY, US: Oxford University Press.

Baddeley, A.D. (2012). Working memory, theories models and controversy. Annual Review of Psychology, 63, 1-29.

Baddeley, A.D., Allen, R. J., \& Hitch, G. J. (2011). Binding in visual working memory: The role of the episodic buffer. Neuropsychologia, 49, 1393-1400.

Baddeley, A.D., Hitch, G. J., \& Allen, R. J. (2009). Working memory and sentence recall. Journal of Memory and Language, 61, 438-456.

Barch, D.M. (2005). The cognitive neuroscience of schizophrenia. Annual Review of Clinical Psychology, 1, 321-353.

Bornstein, M., \& Krinsky, S. (1985). Perception of symmetry in infancy: The saliency of vertical symmetry and the perception of pattern wholes. Journal of Experimental Child Psychology, 39, 1-19.

Bornstein, M., \& Stiles-Davis, J. (1984). Discrimination and memory for symmetry in young children. Developmental Psychology, 20, 639-649.

Brébion, G., David, A. S., Jones, H. M., Ohlsen, R., \& Pilowsky, L. S. (2007). Temporal context discrimination in patients with schizophrenia: Associations with auditory hallucinations and negative symptoms. Neuropsychologia, 45, 817-823.

Brébion, G., Gorman, J. M., Malaspina, D., \& Amador, X. (2005). A model of verbal memory impairments in schizophrenia: Two systems and their associations with underlying cognitive processes and clinical symptoms. Psychological Medicine, 35, 133-142.

Burglen, F., Marczewski, P., Mitchell, K. J., van der Linden, M., Johnson, M. K., Danion, J. M. et al. (2004). Impaired performance in a working memory binding task in patients with schizophrenia. Psychiatry Research, 125, 247-255.

Cowan, N. (2001). The magical number 4 in short-term memory: A reconsideration of mental storage capacity. Behavioral and Brain Sciences, 24, 87-185.

Elsley, J. V., \& Parmentier, F. B. R. (2009). Is verbal-spatial binding in working memory impaired by a concurrent memory load? Quarterly Journal of Experimental Psychology, $62,1696-1705$.

Elvevag, B., Weinberger, D. R., \& Goldberg, T. E. (2001). Short-term memory for serial order in schizophrenia: A detailed examination of error types. Neuropsychology, 15, 128-135.

Fisher, C., Ferdinandes, K., \& Bornstein, M. (1981). The role of symmetry in infant form discrimination. Child Development, 52, 457-462.

Forbes, N. F., Carrick, L. A., McIntosh, A.M., \& Lawrie, S. M. (2009). Working memory in schizophrenia: A meta-analysis. Psychological Medicine, 39, 889-905.

Frazier, P. A., Tix, A. P., \& Barron, K. E. (2004). Testing moderator and mediator effects in counseling psychology research. Journal of Counseling Psychology, 51(1), 115-134.

Gold, J. M., Hahn, B., Zhang, W., Robinson, B.M., Kappenman, E. S., Beck, V. M., et al. (2010). Reduced capacity but spared precision and maintenance of working memory representations in schizophrenia. Archives of General Psychiatry, 67, 570-577.

Gold, J. M., Wilk, C. M., McMahon, R. P., Buchanan, R. W., \& Luck, S. J. (2003). Working memory for visual features and conjunctions in schizophrenia. Journal of Abnormal Psychology, 112, 61-71

Imbo, I., Szmalec, A., \& Vandierendonck, A. (2009). The role of structure on age-related increases in visuo-spatial span performance. Psychology in Belgium, 49, 275-291.

Jaswal, S., \& Logie, R. H. (2013). The contextual interference effect in visual feature binding: What does it say about the role of attention in binding? Quarterly Journal of Experimental Psychology, 66(4), 687-704.

Jefferies, E., Lambon Ralph, M.A., \& Baddeley, A.D. (2004). Automatic and controlled processing in sentence recall: The role of long-term and working memory. Journal of Memory and Language, 51(4), 623-643.

Jiang, Y., Olson, I. R., \& Chun, M. M. (2000). Organization of visual short-term memory. Journal of Experimental Psychology: Learning, Memory, and Cognition, 26, 683-702.

Karlsen, P. J., Allen, R. J., Baddeley, A.D., \& Hitch, G. J. (2010). Binding across space and time in visual working memory. Memory $\mathcal{E}$ ' Cognition, 38, 292-303.
Kay, S. R., Fiszbein, A., \& Opler, L. A. (1987). The Positive and Negative Syndrome Scale 869 (PANSS) for schizophrenia. Schizophrenia Bulletin, 13, 261-276. 870

Kemps, E. (2001). Complexity effects in visuo-spatial working memory: Implications for 871 the role of long-term memory. Memory, 9, 13-27. 872

Knight, R. A., Manoach, D. S., Elliott, D. S., \& Hershenson, M. (2000). Perceptual organiza- 873 tion in schizophrenia: The processing of symmetrical configurations. Journal of 874 Abnormal Psychology, 109(4), 575-587.

Kochan, N. A Valenzuela, M., Slavin, M. J. McCraw, S, Sachdev, PS, \& Breakspear, M. 876 (2011). Impact of load-related neural processes on feature binding in visual working 877 memory. PLoS One, e23960.

Lecerf, T., \& de Ribaupierre, A. (2005). Recognition in a visuospatial memory task: The ef- 879 fect of presentation. European Journal of Cognitive Psychology, 17, 47-75. 880

Lee, J., Folley, B.S., Gore, J. C., \& Park, S. (2008). Origins of spatial working memory deficit 881 in schizophrenia: An event-related fMRI and near-infrared spectroscopy study. PLoS 882 One, e1760.

Lee, J., \& Park, S. (2005). Working memory impairments in schizophrenia: A meta- 884 analysis. Journal of Abnormal Psychology, 114, 599-611. 885

Lewandowsky, S., \& Farrell, S. (2000). A redintegration account of the effects of speech 886 rate, lexicality, and word frequency in immediate serial recall. Psychological 887 Research, 63, 163-173.

Lorsbach, T. C., \& Reimer, J. F. (2005). Feature binding in children and young adults. 889 Journal of Genetic Psychology, 166, 313-327.

Luck, D., Buchy, L., Lepage, M., \& Danion, J. M. (2009). Examining the effects of two factors 891 on working memory maintenance of bound information in schizophrenia. Journal of 892 the International Neuropsychological Society, 15, 597-605.

Luck, D. Foucher, J R Offerlin-Meyer, I. Lepage, M. \& Danion, J. M single and bound features in a working memory task in schizophrenia. Schizophrenia 895 Research, 100, 153-160. 896

Luck, S. J., \& Vogel, E. K. (1997). The capacity of visual working memory for features and 897 conjunctions. Nature, 390, 279-281.

Mitchell, K. J., Johnson, M. K., Raye, C. L., Mather, M., \& D'Esposito, M. (2000). Aging and 899 reflective processes of working memory: Binding and test load deficits. Psychology 900 and Aging, 15, 527-541.

Pieroni, L., Rossi-Arnaud, C., \& Baddeley, A.D. (2011). What can symmetry tell us about 902 working memory? In A. Vandierendonck, \& A. Szmalec (Eds.), Spatial working memo- 903 ry (pp. 145-159). Hove \& NY: Psychology Press.

Raichle, M. E., \& Snyder, A. Z. (2007). A default mode of brain function: A brief history of 905 an evolving idea. Neurolmage, 37, 1083-1090.

Rossi-Arnaud, C., Pieroni, L., \& Baddeley, A.D. (2006). Symmetry and binding in 907 visuo-spatial working memory. Neuroscience, 139, 393-400. 908

Rossi-Arnaud, C., Pieroni, L., Spataro, P., \& Baddeley, A.D. (2012). Working memory in- 909 volvement in symmetry encoding: Differences between vertical, horizontal and diag- 910 onal configurations. Acta Psychologica, 56, 157-166.

Salamé, P., Burglen, F., \& Danion, J. M. (2006). Differential disruptions of working memory 912 components in schizophrenia in an object-location binding task using the suppres- 913 sion paradigm. Journal of the International Neuropsychological Society, 12, 510-518. 914

Schwartz Place, E. J. \& Gilmore, G. C. (1980). Perceptual organization in schizophrenia. 915 Journal of Abnormal Psychology, 89(3), 409-418.

Schweickert, R. (1993). A multinomial processing tree model for degradation and redin- 917 tegration in immediate recall. Memory \& Cognition, 21, 168-175.

Silverstein, S. M., Bakshi, S., Chapman, R. M., \& Nowlis, G. (1998). Perceptual organisation 919 of configural and nonconfigural visual patterns in schizophrenia: Effects of repeated 920 exposure. Cognitive Neuropsychiatry, 3(3), 209-223.

921

Silverstein, S. M. Bakshi, S., Nuernberger, S. Carpinello, K. \& Wilniss, S. (2005). Effects of 922 stimulus structure and target-distracter similarity on the development of visual 923 memory representations in schizophrenia. Cognitive Neuropsychiatry, 10(3), 215-229. 924

Silverstein, S. M., Hatashita-Wong, M., Schenkel, L. S., Wilkniss, S., Kovács, I., Fehér, A., 925 et al. (2006). Reduced top-down influences in contour detection in schizophrenia. 926 Cognitive Neuropsychiatry, 11(2), 112-132.

Silverstein, S. M., Knight, R. A., Schwarzkopf, S. B., West, L. L., Osborn, L. M., \& Kamin, D. 928 (1996). Stimulus configuration and context effects in perceptual organization in 929 schizophrenia. Journal of Abnormal Psychology, 105(3), 410-420. 930

Treisman, A. (2003). The unity of consciousness: Binding, integration, and dissociation. In 931 Axel Cleeremans (Ed.), Consciousness and perceptual binding. New York, NY, US: Ox- 932 ford University Press.

Treisman, A., \& Zhang, W. (2006). Location and binding in visual working memory. 934 Memory \& Cognition, 34, 1704-1719. 935

Uhlhaas, P. J. \& Silverstein, S. M. (2005). Perceptual organization in schizophrenia spec- 936 trum disorders: Empirical research and theoretical implications. Psychological 937 Bulletin, 131(4), 618-632.

Wenderoth, P. (1994). The salience of vertical symmetry. Perception, 23, 221-236. 939

Whitfield-Gabrieli, S., \& Ford, J. M. (2012). Default mode network activity and connectiv- 940 ity in psychopathology. Annual Review of Clinical Psychology, 8, 49-76.

Woodman, G. F., Vecera, S. P., \& Luck, S. J. (2003). Perceptual organization influences visu- 942 al working memory. Psychonomic Bulletin E Review, 10, 80-87. 